\section{Experience Management of Intraoperative Hemorrhage in Laparoscopic Inguinal Hernia Repair}

Sir,

Laparoscopic technique for inguinal hernia has the advantages of small incision, less pain, quick recovery and so on. ${ }^{1}$ Bleeding is one of the common complications of laparoscopic inguinal herniorrhaphy with a incidence of $0.11 \%$; and it can be lifethreatening if not handled properly. ${ }^{2,3}$ Avoiding or correctly managing the hemorrhage during the surgery is very important.

We routinely performed 1,393 laparoscopic inguinal hernia repair in accordance with the guidelines in our hospital. We retrospectively summarised the complications of these cases, and bleeding was found in 48 cases, 10 cases of the inferior epigastric artery hemorrhage, 5 cases of spermatic vessels hemorrhage, 20 cases of the surface vessels of the pubic bone hemorrhage, 12 cases of puncture site hemorrhage, and one case of the iliac vessels hemorrhage. The reasons and corresponding treatment methods of the 48 hemorrhagic cases were investigated and analysed.

Bleeding often occurs in the inferior epigastric artery, spermatic vessels, the surface vessels of the pubic bone, the "death crown", and the iliac vessels, etc. Anatomical variations may exist on the inferior epigastric artery which may lead to bleeding and difficulty of surgical operations on laparoscopic inguinal hernia repair. ${ }^{4}$ In 1,393 cases, abnormal anatomy of inferior epigastric artery was found in 16 cases, including nine cases of the abnormal direction and seven cases of abnormal prolapse. If the puncture point of trocar is located in the left or right lower abdomen, the abnormal inferior epigastric vessels may be damaged when puncturing. So we recommend, the puncture point to be in the lower abdomen ( $1 / 3$ and $2 / 3$ of the line of umbilicus and pubic symphysis). Besides, the forceps can be used to lift up the inferior epigastric artery to fully expose the operative field.

Another reason for hemorrhage in laparoscopic inguinal hernia repair was the surgeon's unfamiliarity with anatomy, which caused improper procedure during the operation. According to the reporters, the learning curve of the laparoscopic inguinal hernia repair was 13 cases. Compared to TEP, the learning curve of TAPP was shortened ( 14 cases vs. 13 cases). ${ }^{5}$

Once serious bleeding appears, due to some limitations of the laparoscopic technique, such as the small operating space, or limited use of instrument, which may lead to the failure of surgery, even hemorrhagic shock and death. If the spermatic vessels or the surface vessels of the pubic bone are bleeding, we can use the gauze compression packing hemostasis, or electrocoagulation for hemostasis (Figure $1 \mathrm{~A}$ ). If active hemorrhage is caused by the inferior epigastric artery injury, operating forceps should be quickly clipped the broken blood

vessels to stop bleeding and stem the bleeding with electrocautery or occlude it with absorbable clamps (Figure 1B). If it bleeds heavily or operative fields have been influenced, traditional open surgery should be performed. If there is 'death crown' or the iliac vascular injury happens, traditional open surgery should immediately be implemented.

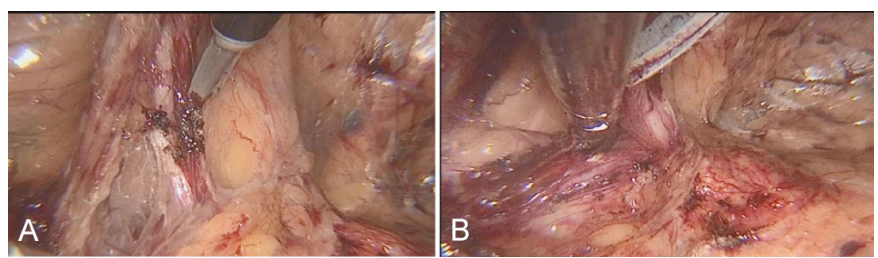

Figure 1: Management of intraoperative hemorrhage. A: Electrocoagulation for hemostasis; B: Operating forceps and electrocautery are used for active hemorrhage caused by the inferior epigastric artery injury.

\section{FUNDING:}

This study was supported by National Natural Science Foundation China (81602613).

\section{CONFLICT OF INTEREST:}

Authors declared no conflict of interest.

\section{AUTHORS' CONTRIBUTION:}

JX, XZ: Responsible for the conception and design of the work, or revising it critically for important intellectual content, or the analysis of data for the work.

CG: Final approval of the version to be published and agreed to be accountable for all aspects of the work.

\section{REFERENCES}

1. Zhu X, Cao H, Ma Y, Yuan A, Wu X, Miao Y, et al. Totally extraperitoneal laparoscopic hernioplasty versus open extraperitoneal approach for inguinal hernia repair: A metaanalysis of outcomes of our current knowledge. Surgeon 2014; 12(2):94-105. doi: 10.1016/j.surge.2013.11.018.

2. Grant AM \& EU Hernia Trialists Collaboration. Laparoscopic versus open groin hernia repair: Meta-analysis of randomised trials based on individual patient data. Hernia 2002; 6:2-10. doi: 10.1007/s10029-002-0050-8.

3. Yasuda T, Matsuda A, Miyashita M, Matsumoto S, Sakurazawa N, Kawano $\mathrm{Y}$, et al. Life-threatening hemorrhage from the corona mortis after laparoscopic inguinal hernia repair: Report of a case. Asian J Endosc Surg 2018; 11(2):169-72. doi: 10.1111/ases.12416.

4. Altalalwah W. The inferior epigastric artery: Anatomical study and clinical significance. Int J Morphol 2017; 35(1): 7-11.

5. Bansal VK, Krishna A, Misra MC, Kumar S. Learning curve in laparoscopic inguinal hernia repair: Experience at a tertiary care centre. Indian J Surg 2016; 78(3):197-202. doi: 10.1007/s12262-015-1341-5. 
Jiaming Xie, Xiaofei Zheng and Chunwei Gu

Department of General Surgery, The Second Affiliated

Hospital of Soochow University, China

Correspondence to: Dr. Chunwei Gu, Department of General
Surgery, The Second Affiliated Hospital of Soochow University, Suzhou, China

E-mail: zheng20175233064@163.com

Received: October 30, 2019; Revised: January 29, 2020;

Accepted: February 11, 2020

DOI: https://doi.org/10.29271/jcpsp.2020.11.1232 